\title{
Emission from Silica Hybrid Containing RGB Fluorescent Conjugated Polymers
}

\author{
Yuya Sugiura $^{\mathrm{a}}$, Masashi Shoyama ${ }^{\mathrm{b}}$, Koji Inoue $^{\mathrm{b}}$, Takahiro Uno ${ }^{\mathrm{a}}$, Takahito Itoh ${ }^{\mathrm{a}}$, \\ Masataka Kubo ${ }^{\mathrm{a}}\left(\boldsymbol{\varpi}^{\prime}\right)$ \\ ${ }^{a}$ Division of Chemistry for Materials, Graduate School of Engineering, Mie University, Tsu \\ 514-8507, Japan \\ ${ }^{\mathrm{b}}$ Industrial Research Division, Mie Science and Technology Promotion Center, Tsu 514-0819, \\ Japan \\ E-mail: kubo@chem.mie-u.ac.jp, Fax: +81-59-231-9471
}

Received: 31 May 2006 / Accepted: 20 July 2006

Published online: 8 August 2006 - @ S Springer-Verlag 2006

\section{Summary}

Red, green and blue fluorescent poly(arylene vinylene)s were prepared by Wittig reaction. Polycondensation of tetraethoxysilane (TEOS) was carried out in the presence of a mixed solution of these polymers to give a silica hybrid in which RGB fluorescent polymers were immobilized without phase separation. White light emission was observed from the ternary polymer blend/silica hybrid.

\section{Introduction}

Organic/inorganic hybrid materials have attracted great interest as new materials which combine the advantage of organic molecule (flexibility, processability, ductility) and the inorganic material (rigidity, thermal stability) $[1,2]$. Since $\pi$ conjugated polymers have been utilized in the field of optoelectronic applications $[3,4]$, their hybrids with silica can provide new materials that have interesting properties. In a previous study, we carried out acid-catalyzed polycondensation of TEOS in the presence of poly[2-methoxy-5-(2-ethylhexyloxy)-1,4-phenylene vinylene) (MEH-PPV) prepared by Wittig reaction to obtain homogeneous MEHPPV/silica hybrid [5]. We are interested in immobilizing RGB fluorescent polymers in silica matrix which serves to separate the light-emitting domains. Each molecule emits in an isolated dispersed state to give white light emission due to the suppression of energy transfer between emitting polymers. Recently, several research groups studied emission from polymer/polymer blends composed of conjugated polymer and an inert polymer such as polystyrene or poly(methyl methacrylate) [6-8]. In this paper we report polycondensation of TEOS in the presence of a mixed solution of RGB fluorescent polymers to obtain a ternary polymer blend/silica hybrid and its optical property. Poly[(9,9-dioctylfluorenyl-2,7-diyl vinylene)-alt-(anthracene-9,10-diyl vinylene)] (1), poly[(9,9-dioctylfluorenyl-2,7-diyl vinylene)-alt-(2-methoxy-5-(2ethylhexyloxy)-1,4-phenylene vinylene)] (2) and poly[(2,5-bis(trimethylsilyl)-1,4phenylene vinylene)-alt-(1,2-phenylene vinylene)] (3) have been chosen as red, green and blue $\pi$-conjugated fluorescent polymers, respectively (Scheme 1). 


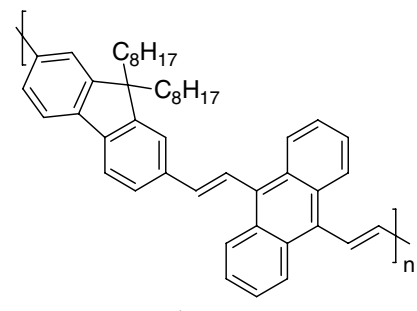

1

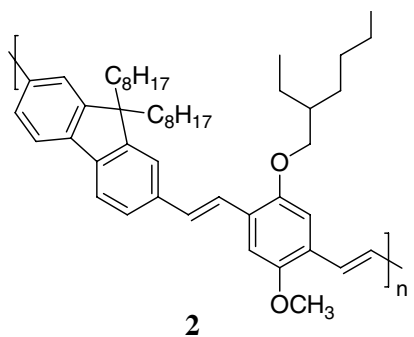<smiles>C[SiH3]c1cc(/C=C/c2ccccc2/C=C\C(C)(C)C)c([SiH2])cc1C(C)(C)C</smiles>

3

Scheme 1. Red (1), green (2) and blue (3) fluorescent $\pi$-conjugated polymers

\section{Experimental}

\section{Materials}

Poly[(2,5-bis(trimethylsilyl)-1,4-phenylene vinylene)-alt-(1,2-phenylene vinylene)] (3) was prepared according to the reported method [9]. TEOS was distilled under reduced pressure. Solvents and other reagents were purified by usual methods.

\section{2,7-Bis(triphenylphosphoniomethyl)-9,9-dioctylfluorene dibromide}

A mixture of 2,7-bis(bromomethyl)-9,9-dioctylfluorene [10] (12.0 g, $21 \mathrm{mmol}$ ), triphenylphosphine $(16.5 \mathrm{~g}, 63 \mathrm{mmol})$ and $200 \mathrm{~mL}$ of dimethylformamide (DMF) was stirred at $160^{\circ} \mathrm{C}$ for $12 \mathrm{~h}$. The reaction mixture was cooled to room temperature and poured into ether to precipitate the white powder. It was filtered and recrystallized from ethanol to give $13.0 \mathrm{~g}(64 \%)$ of 2,7-bis(triphenylphosphoniomethyl)-9,9dioctylfluorene dibromide as white plates. $\mathrm{mp}>250{ }^{\circ} \mathrm{C} .{ }^{1} \mathrm{H} \mathrm{NMR}\left(\mathrm{CDCl}_{3}, \delta\right.$, ppm): 7.8-7.6 (m, 30H), 7.38 (d, $J=7.9 \mathrm{~Hz}, 2 \mathrm{H}), 7.06(\mathrm{~d}, J=7.9 \mathrm{~Hz}, 2 \mathrm{H}), 6.97$ (s, 2H), 5.46 $(\mathrm{d}, J=14.5 \mathrm{~Hz}, 4 \mathrm{H}), 1.5-1.4(\mathrm{~m}, 4 \mathrm{H}), 1.3-1.1(\mathrm{~m}, 10 \mathrm{H}), 0.83(\mathrm{t}, J=6.9 \mathrm{~Hz}, 6 \mathrm{H}), 0.20$ (brs, $4 \mathrm{H}) .{ }^{13} \mathrm{C}$ NMR $\left(\mathrm{CDCl}_{3}, \delta, \mathrm{ppm}\right): 151.1,140.3,134.9,134.1,130.6,130.0,125.9$, 125.0, 120.2, 118.1, 54.7, 39.7, 31.6, 30.9, 29.8, 29.3, 29.2, 23.8, 22.4, 13.9. IR (KBr, $\mathrm{cm}^{-1}$ ): 2925 (s), 2850 (s), $1470(\mathrm{~m}), 1450$ (m), 1113 (m), 846 (w), 747 (s). Anal: Calcd for $\mathrm{C}_{67} \mathrm{H}_{74} \mathrm{Br}_{2} \mathrm{P}_{2}: 73.09 \% \mathrm{C} ; 6.77 \% \mathrm{H}$. Found: $73.32 \% \mathrm{C} ; 6.68 \% \mathrm{H}$.

\section{Poly[(9,9-dioctylfluorenyl-2,7-diyl vinylene)-alt-(anthracene-9,10-diyl vinylene)] (1)}

To a stirred solution of an equimolar amount of 2,7-bis(triphenylphosphoniomethyl)9,9-dioctylfluorene dibromide $(661 \mathrm{mg}, \quad 0.60 \mathrm{mmol})$ and anthracene-9,10dicarbaldehyde [11] (141 mg, $0.60 \mathrm{mmol})$ in a mixture of $4 \mathrm{~mL}$ of tetrahydrofuran (THF) and $4 \mathrm{~mL}$ of dimethylsulfoxide (DMSO) was added dropwise $0.84 \mathrm{~mL}$ of $1.6 \mathrm{M} n$-butyllithium in hexane $(1.3 \mathrm{mmol})$ under nitrogen. The reaction mixture was stirred for $24 \mathrm{~h}$ and poured into methanol to precipitate the polymer. It was washed well with methanol to give $285 \mathrm{mg}(77 \%)$ of $\mathbf{1}$ as an orange powder. GPC (THF eluent, polystyrene standard): $M_{\mathrm{n}}=6400 \mathrm{Da}$.

Poly[(9,9-dioctylfluorenyl-2,7-diyl vinylene)-alt-(2-(2-ethylhexyloxy)-5-methoxy-1,4phenylene vinylene)] (2)

To a stirred solution of an equimolar amount of 2,7-bis(triphenylphosphoniomethyl)9,9-dioctylfluorene dibromide (661 $\mathrm{mg}, \quad 0.60 \mathrm{mmol})$ and 2-methoxy-5-(2- 
ethylhexyloxy)benzene-1,4-dicarbaldehyde [12] (175 $\mathrm{mg}, 0.60 \mathrm{mmol})$ in a mixture of $8 \mathrm{~mL}$ of THF and $6 \mathrm{~mL}$ of DMSO was added dropwise $0.84 \mathrm{~mL}$ of $1.6 \mathrm{M} \mathrm{n}$ butyllithium in hexane $(1.3 \mathrm{mmol})$ under nitrogen. The reaction mixture was stirred for $24 \mathrm{~h}$ and poured into methanol to precipitate the polymer. It was washed well with methanol to give $260 \mathrm{mg}(66 \%)$ of $\mathbf{2}$ as a yellow powder. GPC (THF eluent, polystyrene standard): $M_{\mathrm{n}}=6500 \mathrm{Da}$.

\section{Silica Hybrid Containing RGB Fluorescent Polymers}

A mixture of polymer $1(2.0 \mathrm{mg})$, polymer $2(1.0 \mathrm{mg})$, polymer $3(3.0 \mathrm{mg})$, TEOS (4 $\mathrm{mL})$, THF $(5 \mathrm{~mL}), 1 \mathrm{M}$ hydrochloric acid $(1 \mathrm{~mL})$, and DMSO $(1 \mathrm{~mL})$ was stirred at room temperature in a polypropyrene test tube $(15 \mathrm{~mm}$ in diameter and $150 \mathrm{~mm}$ in length). The tube was set in an oven and heated at $60{ }^{\circ} \mathrm{C}$ for $48 \mathrm{~h}$. The temperature was gradually increased to $100{ }^{\circ} \mathrm{C}$ and allowed to stand for additional $48 \mathrm{~h}$. Finally, the obtained gel was dried under vacuum to give a transparent orange solid of cylindrical shape.

\section{Measurements}

${ }^{1} \mathrm{H}$ and ${ }^{13} \mathrm{C}$ NMR spectra were recorded at room temperature on a JEOL EX-270 nuclear magnetic resonance at 270 and $67.5 \mathrm{MHz}$, respectively. Samples were dissolved in $\mathrm{CDCl}_{3}$ and tetramethylsilane (TMS) was added as the internal standard. Infrared spectra were recorded on a Jasco IR-700 infrared spectrophotometer. Gel permeation chromatography (GPC) was carried out on a Tosoh HLC-8020 chromatograph equipped with polystyrene gel columns (Tosoh G2500H + G3000H; exclusion limit $=6 \times 10^{4} ; 300 \times 7.5 \mathrm{~mm}$ ) and refractive/ultraviolet dual mode detectors. THF was used as the eluent at a flow rate of $1.0 \mathrm{~mL} / \mathrm{min}$. The calibration curves for GPC analysis were obtained using polystyrene standards. Photoluminescence spectra were recorded on a HITACHI F-4500 fluorescence spectrophotometer with exciting wavelength of $365 \mathrm{~nm}$. The Commission Internationale de I'Eclairage (CIE) coordinate of the emission was determined by HAMAMATSU PMA-11 photonic multi channel analyzer. Elemental analysis was performed on Yanaco MT-5 CHN CORDER.

\section{Results and discussion}

\section{Polymer Synthesis}

Preliminary experiment in our laboratory showed that acid-catalyzed polycondensation of TEOS in the presence of commercial available poly[ $(9,9-$ dihexylfluorenyl-2,7-diylvinylene)-co-(2-methoxy-5-(2-ethylhexyloxy)-1,4-phenylene vinylene)] (Aldrich) did not give homogeneous hybrid. The organic polymer precipitated during the sol-gel process. This is due to the lack of any chemical or physical interactions between organic polymer and silica. Thus, three fluorescent poly(arylene vinylene)s were prepared utilizing Wittig reaction between diphosphonium and dialdehyde compounds. Statistically, a polymer chain prepared by this method has a phosphonium salt moiety at the chain end. The polar phosphonium salt group enhances the compatibility between the polymer and silicate during the sol-gel process due to the ionic interaction. 
Firstly, red fluorescent polymer 1 was prepared by the reaction of 2,7bis(triphenylphosphoniomethyl)-9,9-dioctylfluorene dibromide and anthracene-9,10dicarbaldehyde according to Scheme 2. Figure 1 shows ${ }^{1} \mathrm{H}$ NMR spectrum of $\mathbf{1}$. The peaks due to the terminal structures are clearly observed. The peak at $5.5 \mathrm{ppm}$ corresponds to the methylene protons arising from the triphenylphosphoniomethyl groups. The peaks around $11.5 \mathrm{ppm}$ are assignable to the terminal aldehyde groups.
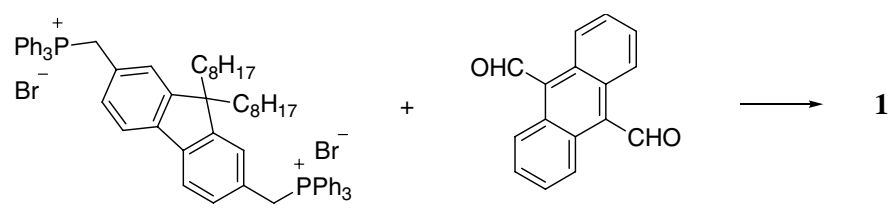

Scheme 2. Preparation of red fluorescent polymer 1

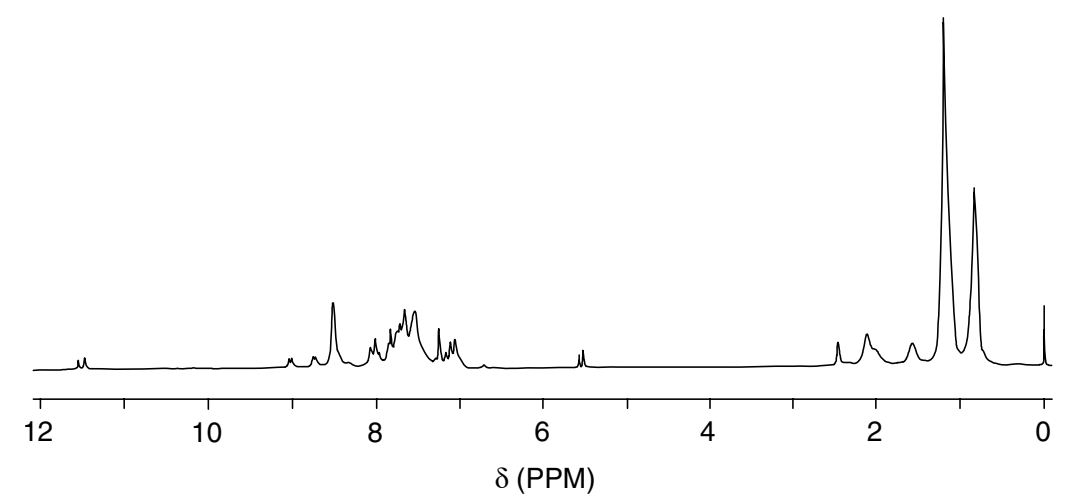

Figure 1. ${ }^{1} \mathrm{H}$ NMR spectrum of red fluorescent polymer 1 prepared by Wittig method

Secondly, Wittig reaction of 2,7-bis(triphenylphosphoniomethyl)-9,9-dioctylfluorene dibromide with 2-methoxy-5-(2-ethylhexyloxy)benzene-1,4-dicarbaldehyde was carried out to obtain green fluorescent polymer 2 according to Scheme 3. Its ${ }^{1} \mathrm{H}$ NMR spectrum exhibits absorption peaks due to the terminal structure as shown in Figure 2.
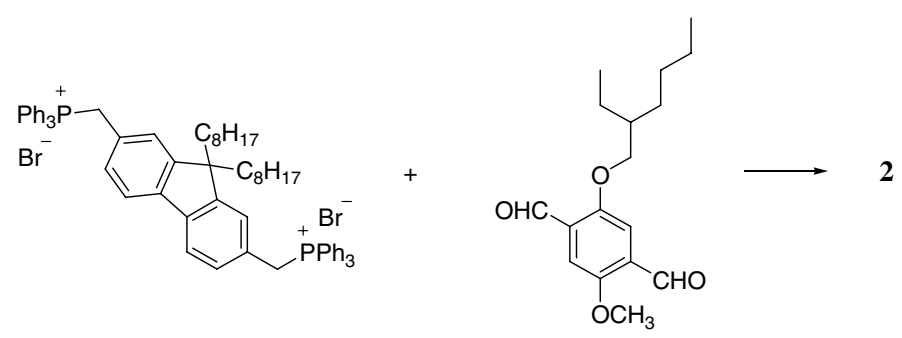

Scheme 3. Preparation of green fluorescent polymer 2

Finally, blue fluorescent polymer 3 was prepared by Wittig reaction between 2,5bis(trimethylsilyl)-1,4-xylenebis(triphenylphosphonium bromide) and phthalaldehyde according to the reported procedure (Scheme 4). The polymer was purified by using 
a Soxhlet extractor in methanol. The $M_{\mathrm{n}}$ of $\mathbf{3}$ was determined by GPC to be $8000 \mathrm{Da}$ as polystyrene standard.

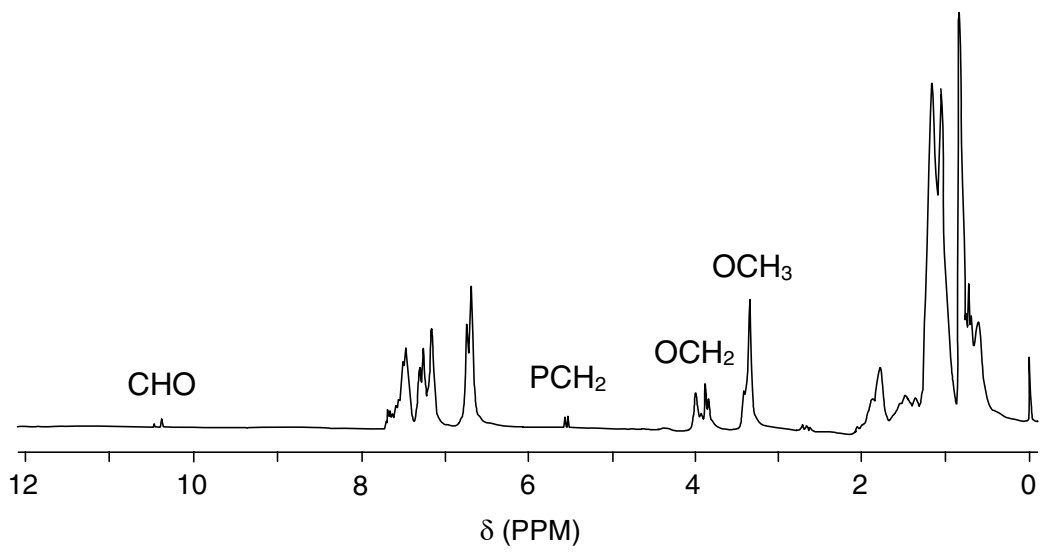

Figure 2. ${ }^{1} \mathrm{H}$ NMR spectrum of green fluorescent polymer 2 prepared by Wittig method

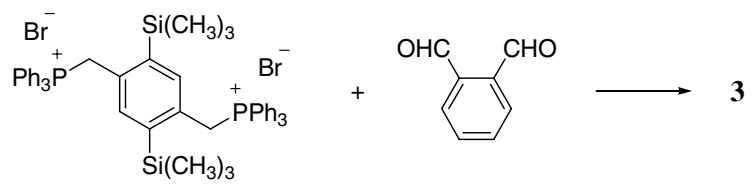

Scheme 4. Preparation of blue fluorescent polymer 3

\section{Sol-Gel Method}

The preparation of silica hybrid was carried out by the acid-catalyzed hydrolysis and polycondensation of TEOS in the presence of a mixed solution of $\mathbf{1 , 2}$ and $\mathbf{3}$ in THF according to the previously reported procedure [5]. As a representative example, a silica hybrid with a weight ratio of $2: 1: 3$ between $\mathbf{1}, \mathbf{2}$ and $\mathbf{3}$ was investigated. The obtained hybrid was a transparent solid without polymer precipitation, indicating a homogeneous hybrid. Addition of high boiling point solvent such as DMSO was effective in order to obtain a crack-free hybrid. For comparison, polymer blend film was made by casting of THF solution of the three polymers onto a quartz plate $(\mathbf{1} / \mathbf{2} / \mathbf{3}$ blend, $2: 1: 3$ by weight).

\section{Optical Property}

Figure 3 shows photoluminescence spectra of $\mathbf{1}, \mathbf{2}$ and $\mathbf{3}$ in THF solution. The emission maxima of $\mathbf{1}$ (dotted line), $\mathbf{2}$ (dashed line) and $\mathbf{3}$ (solid line) are 565, 515 and $470 \mathrm{~nm}$, respectively.

Figure $4 \mathrm{a}$ shows the photoluminescence spectra of the silica hybrid (solid line) and the blend film (dotted line). The blend film gave narrower spectrum. On the other hand, the photoluminescence spectrum of the silica hybrid exhibited a broad emission from 450 to $600 \mathrm{~nm}$. This means molecular-sized small domains independently emit in silica matrix. 


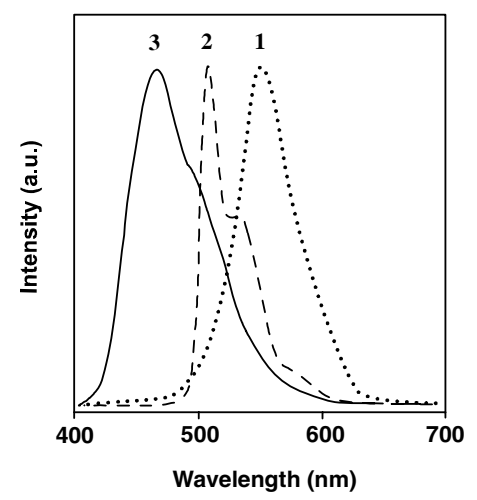

Figure 3. Photoluminescence spectra of $\mathbf{1}, \mathbf{2}$ and $\mathbf{3}$ in THF solution

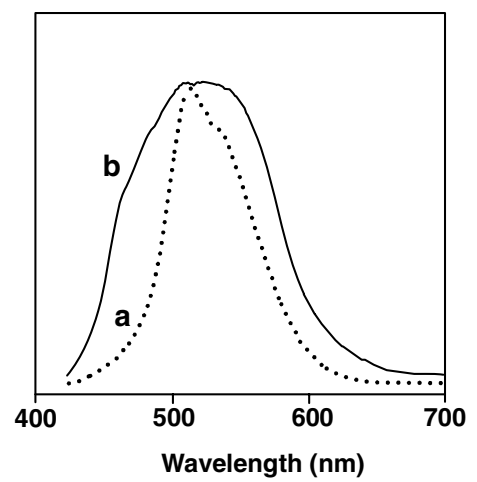

Figure 4. Photoluminescence spectra of (a) blend film and (b) silica hybrid

Figure 5 shows CIE diagram for solution of 1, 2 and 3, blend film and silica hybrid. The coordinates of the emission from $\mathbf{1}, \mathbf{2}$ and $\mathbf{3}$ in solution state were $(0.44,0.47)$, $(0.32,0.61)$ and $(0.17,0.28)$, respectively. The emission from the blend film $(0.47$, 0.48 ) corresponds to orange-yellow color probably due to the energy transfer between polymers. The silica hybrid containing RGB polymers gave coordinates of $(0.30$, 0.42). To the eye, the emission from the silica hybrid appeared to be white.

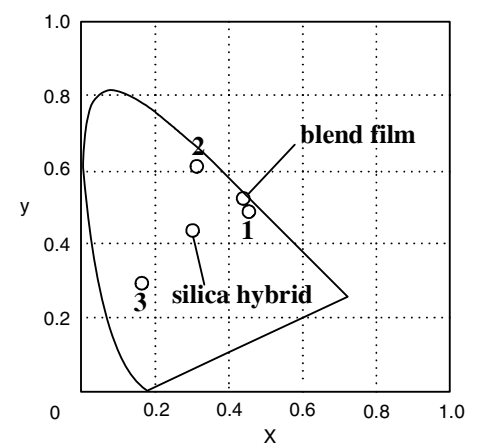

Figure 5. CIE chromaticity diagram showing the color of the emission from 1, 2 and 3 in THF solution, blend film and silica hybrid 


\section{Conclusions}

In conclusion, red, green and blue fluorescent poly(arylene vinylene)s were prepared by Wittig reaction. Polycondensation of TEOS was carried out in the presence of a mixed solution of these polymers to give a silica hybrid in which RGB fluorescent polymers were immobilized without phase separation. The ternary polymer blend/silica hybrid gave a broad emission. A proper combination of emitting polymers will give the necessary spectral distribution because each polymer is homogeneously immobilized in an inert silica matrix.

Acknowledgement. This study has partly been supported by Co-operation of Innovative Technology and Advanced Research in Evolution Area Project (Ministry of Education, Culture, Sports, Science and Technology).

\section{References}

1. Novak BM (1993) Adv Mater 5:422.

2. Wen J, Wilkes GL (1996) Chem Mater 8:1667.

3. Bredas JL, Silbey R (Eds.) (1991) Conjugated Polymers, Kluwer Akademic Publishers, Dordrecht, The Netherlands.

4. Farchioni R, Grosso G (Eds.) (2001) Organic Electronic Materials, Springer, New York.

5. Kubo M, Takimoto C, Minami Y, Uno T, Itoh T, Shoyama M (2005) Macromolecules 38:7314.

6. Granström M, Inganäs O (1996) Appl Phys Lett 68:147.

7. He G, Li Y, Liu J, Yang Y (2002) Appl Phys Lett 80:4247.

8. Ananthakrishnan N, Padmanaban, G, Ramakrishnan S, Reynolds JR (2005) Macromolecules 38:7660.

9. Ahn T, Jang MS, Shim H-K, Hwang D-H, Zyung T (1999) Macromolecules 32:3279.

10. Liu B, Yu W-L, Pei J, Liu S-Y, Lai Y-H, Huang W (2001) Macromolecules 34:7932.

11. Lin Y-I, Lang SA, Seifert CM, Child RG, Morton GO, Fabio PF (1979) J Org Chem 44:4701.

12. Park LS, Han YS, Hwang JS, Kim SD (2000) J Polym Sci Part A Polym Chem 38:3137. 